Research Article

\title{
Investigation of Virulence Genes of Staphylococcus aureus Isolated from Sterile Body Fluid Samples and Their Correlation with Clinical Symptoms and Outcomes
}

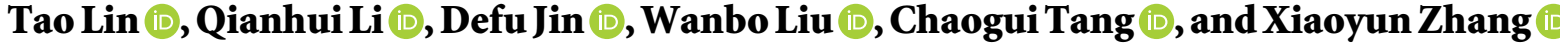 \\ Department of Medical Laboratory, The Affiliated Huaian No. 1 People's Hospital of Nanjing Medical University, \\ Huanghe West Road 1, Huai'an, Jiangsu 223300, China \\ Correspondence should be addressed to Xiaoyun Zhang; xyzhang2009@126.com
}

Received 29 September 2021; Accepted 15 December 2021; Published 26 December 2021

Academic Editor: Muhammad Abu Bakr Shabbir

Copyright $\odot 2021$ Tao Lin et al. This is an open access article distributed under the Creative Commons Attribution License, which permits unrestricted use, distribution, and reproduction in any medium, provided the original work is properly cited.

\begin{abstract}
Staphylococcus aureus is the major pathogen causing nosocomial human infections and produces a variety of virulence factors that contribute to its ability to colonize and cause diseases. This study was conducted to investigate the virulence genes in $S$. aureus isolated from sterile body fluid samples and their correlation with clinical symptoms and outcomes. The VITEK $2^{\circledR}$ Compact system was used to perform biochemical identification and antimicrobial susceptibility tests on $33 \mathrm{~S}$. aureus isolates. Virulence genes were amplified using multiplex PCR. The virulence gene patterns were analyzed by systematic cluster analysis. The frequency of methicillin-resistant $S$. aureus was $45.45 \%$, and 17 virulence genes were identified. Genes encoding hemolysins showed high frequencies. The frequencies of $h l a, h l b, h l d$, and $h l g B$ were $93.94 \%$ and that of the $l u k-F / S-P V$ was $21.21 \%$. Except for the frequency of $\operatorname{splB}(51.52 \%)$, the remaining genes encoding invasive proteases showed frequencies greater than $81.82 \%$. Among the patients, $100.00 \%$ had undergone invasive medical procedures and $24.00 \%$ had been treated with more than three types of antibiotic drugs. Invasive medical procedures are the main causes of infection. Resistance to antibiotic drugs and the status of carrying virulence genes were highly related to clinical symptoms and outcomes.
\end{abstract}

\section{Introduction}

Staphylococcus aureus is a common opportunistic pathogen that causes large numbers of infections because of the presence of its virulence factors and high resistance to most antibacterial drugs [1]. It is the leading cause of lethality in hospital- and community-acquired infections [2]. The relationship between $S$. aureus colonization and pathogenicity and human diseases has been extensively investigated [3, 4]. Approximately 500,000 S. aureus-related infections occur annually in the United States [5]. Staphylococcus aureus is also one of the most common and fatal causes of blood infection, the incidence of which is increasing [6].

Different $S$. aureus strains carry different virulence factors and cause varying pathogenic characteristics, resulting in different diseases [7-11]. Recent clinical research has focused on the relationship between virulence factors, bacterial evolution, and host factors [12, 13]. Virulence factors in S. aureus include hemolysin, leukocidin, invasive proteases, hyaluronidase, lipase, nuclease, and staphylokinase. Hemolysin plays an important role in colonization and pathogenicity $[14,15]$ by creating holes in the host cell membrane, enabling water and other toxic factors to flow into the host cell and cause cell swelling, rupture, and necrosis $[16,17]$. Some domestic scholars showed that hemolysin also has proinflammatory and apoptotic effects [13]. Foreign research revealed that hemolysin can also cause severe pneumonia. The genes encoding hemolysin include $h l a, h l b, h l d, h l g A, h l g B$, and $h l g C$. Leukocidin exerts destructive effects on host blood cells (including leukocytes), skin, and mucosal cells, leading to inflammatory reactions [18]. The genes encoding leukocidin include $l u k-F / S-P V$, lukE, lukM, psm-mec, and psm- $\alpha$. Invasive toxins include invasive proteases, staphylokinase, hyaluronidase, lipases, and nucleases, which degrade a variety of macromolecules in host tissue cells, allowing inflammation to spread to deeper 
parts of the body. Staphylokinase, also known as plasmin, induces the spread of fibrinolytic bacteria. Hyaluronidase can lyse the extracellular matrix to induce inflammation spreading. Lipase expression products can degrade fats and oils, which are beneficial for the colonization of bacterial strains [19]. Nucleases produced by $S$. aureus can hydrolyze nucleic acids (including DNA and RNA) in host cells. The genes encoding invasive toxins include $s p A$, $s p l B, s p l C, s p l B$, sak, hys A, lip, and nuc.

We have been continuously monitoring the drug resistance genes and virulence genes of $S$. aureus isolated from clinical samples at the Affiliated Huaian No.1 People's Hospital of Nanjing Medical University since 2013 [20-22]. In this study, we investigated the virulence genes of 33 $S$. aureus strains isolated from sterile body fluid samples of patients in our hospital and investigated the relationships between methicillin-resistant $S$. aureus (MRSA), invasiveness, clinical symptoms, and outcomes.

\section{Materials and Methods}

2.1. Bacterial Identification and Antibiotic Susceptibility. All 33 S. aureus strains were isolated from sterile body fluid specimens of inpatients at the Affiliated Huaian No. 1 People's Hospital of Nanjing Medical University from April 2015 to November 2019 and stored at $-80{ }^{\circ} \mathrm{C}$. Blood samples accounted for $84.85 \%(28 / 33)$ of the total samples, and pleural effusion samples accounted for $18.18 \%$ (5/33) of the total samples. The strains were isolated, cultured, and identified in strict accordance with the "National Clinical Laboratory Operating Procedures." All isolates were initially identified based on the colony morphology, Gram staining, and coagulase tests performed at the clinical microbiology laboratory. The results were confirmed using an automated VITEK $2^{\circledR}$ Compact system (bioMérieux, Marcy-l'Étoile, France) using a GP identification card. Antimicrobial susceptibility tests were performed using the automated VITEK $2^{\circledR}$ Compact system with the AST-P639 card, and strains showing resistance to nonsusceptibility to at least one agent in three or more antimicrobial categories were classified as multidrug resistant. Staphylococcus aureus ATCC29213 was used as a quality control strain.

2.2. DNA Extraction. A pure culture colony on blood agar plates was transferred to a $0.5 \mathrm{~mL}$ centrifuge tube containing $200 \mu \mathrm{L}$ proteinase $\mathrm{K}(200 \mathrm{ng} / \mathrm{mL})$. After one cycle of freezing-thawing $\left(56^{\circ} \mathrm{C}\right.$ for $2 \mathrm{~h}, 95^{\circ} \mathrm{C}$ for $10 \mathrm{~min}$ ), the tubes were centrifuged for $30 \mathrm{~s}$ at $24,400 \times \mathrm{g}$ to sediment the impurities. The supernatant was stored at $-20{ }^{\circ} \mathrm{C}$ until use as a template for PCR amplification.

2.3. Virulence Gene Detection. A multiplex PCR assay was used to detect virulence genes. Primers were designed based on previous studies $[23,24]$ and are listed in Table 1 . The primers were synthesized by Nanjing Qingke Biotechnology Company (Nanjing, China). The multiplex PCR sample contained $2 \mu \mathrm{L}$ of DNA template in a $50 \mu \mathrm{L}$ final reaction mixture of $45 \mu \mathrm{L}$ of gold (green) and $10 \mu \mathrm{M}$ upstream and downstream primers. The reaction mixtures were amplified using a thermocycler (Eppendorf, Hamburg, Germany) under the following conditions: initial denaturation at $98^{\circ} \mathrm{C}$ for $2 \mathrm{~min}$, followed by 35 cycles of denaturation at $98{ }^{\circ} \mathrm{C}$ for $10 \mathrm{~s}$, annealing at $72{ }^{\circ} \mathrm{C}$ for $20 \mathrm{~s}$, and extension at $72{ }^{\circ} \mathrm{C}$ for $1 \mathrm{~min}$, and a final extension step at $72{ }^{\circ} \mathrm{C}$ for $1 \mathrm{~min}$. The PCR products were separated using electrophoresis on a $1.5 \%$ agarose gel containing DNA safe stain and visualized using a UV transilluminator. GeneRuler ${ }^{\mathrm{TM}} 100 \mathrm{bp}$ DNA Ladder was used as a molecular weight marker.

2.4. Statistical Analysis. Statistical analyses were performed using the chi-square or Fisher's exact test using SPSS software 16 (SPSS, Inc., Chicago, IL, USA). The results were considered statistically significant when the $P$ value was less than 0.05 .

\section{Results and Discussion}

3.1. Antimicrobial Susceptibility. The antimicrobial resistance profiles for $S$. aureus isolates are listed in Table 2. The resistance rate of $33 \mathrm{~S}$. aureus strains to penicillin $\mathrm{G}$ was $100 \%(33 / 33)$, to oxacillin was $45.45 \%(15 / 33)$, to erythromycin was $84.85 \%$ (28/33), to ciprofloxacin was $72.73 \%$ (24/ $33)$, and to chloramphenicol was $6.06 \%(2 / 33)$. No strains were resistant to vancomycin, teicoplanin, linezolid, or tigecycline. The frequency of MRSA infection was $45.45 \%$ $(15 / 33)$.

3.2. Distribution of Virulence Genes among Methicillin-Susceptible S. aureus (MSSA) and MRSA Strains. Seventeen virulence genes were detected among the $33 \mathrm{~S}$. aureus strains and are shown in Table 3. The frequency of hla and $h l b$ was $96.97 \%(32 / 33)$, which was the highest value. The frequency of $l u k E$ was $51.52 \%(17 / 33)$, while that of $l u k-F / S-P V$ and lukM was $21.21 \%$ (7/33), which was the lowest value. The frequency of $s p l B$ was $51.52 \%(17 / 33)$, and the remaining genes encoding protease were all above $81.2 \%(27 / 33)$. The frequencies of hys A, sak, lip, and nuc were $30.30 \%(10 / 33)$, $69.70 \%(23 / 33), 84.85 \%$ (28/33), and $96.97 \%(32 / 33)$, respectively.

The frequency of $h l a, h l b, h l g B$, and $l i p$ in the MRSA strains was $100 \%(15 / 15)$, and that of $h \lg C$ was $6.67 \%(1 / 15)$. $L u k-F / S-P V$ was not detected in the MRSA strains. The frequency of $h l a, h l b, h l d, h l g B$, and lip among the 18 methicillin-susceptible $S$. aureus (MSSA) strains was $94.44 \%$ (17/18), which was the highest value. $l u k-F / S-P V$ showed the lowest frequency of $5.56 \%(1 / 18)$. The frequencies of $l u k-F / S$ $P V$ and $s s p B$ in the MSSA strains were $5.56 \%(1 / 18)$ and $16.67 \%(3 / 18)$, respectively, which were significantly lower than those in MRSA strains $(P<0.05)$. The frequency of $h l g C$ in the MSSA strains was $83.33 \%(15 / 18)$, which was significantly higher than that in the MRSA strains $(P<0.05)$.

3.3. Virulence Gene Patterns of 33 S. aureus Strains. Twenty-one gene patterns were found among the 33 S. aureus strains and are shown in Table 4. Strain nos. 19, 20, 
TABLE 1: Virulence factors, genes, primer sequences, and product length.

\begin{tabular}{|c|c|c|c|c|}
\hline Virulence factors & Genes & Primer name & Sequence $\left(5^{\prime}-3^{\prime}\right)$ & Product length $(\mathrm{bp})$ \\
\hline \multirow{11}{*}{ Hemolysin } & hla & $\begin{array}{l}\text { hla-F } \\
\text { hla-R }\end{array}$ & $\begin{array}{c}\text { ATGAAAACACGTATAGTCAGCTCAGTAACAAC } \\
\text { TTAATTTGTCATTTCTTCTTTTTCCCAATCGA }\end{array}$ & 960 \\
\hline & & $h l b-\mathrm{F}$ & AAATTAGAGCTGAACAAATGAAAGAAATCAG & \multirow{2}{*}{825} \\
\hline & nlb & $h l b-\mathrm{R}$ & TTTCGCAATTGAATTTGATTGAGGGTCCC & \\
\hline & hld & hld-F & ATGGCACAAGATATCATTTCAACAATCAGTG & \multirow{2}{*}{81} \\
\hline & min & hld-R & TTATTTTTTAGTGAATTTGTTCACTGTGTCG & \\
\hline & & $h \lg A-\mathrm{F}$ & ATGATTAAAAATAAAATATTAACAGCAACTTTAGCAGTT & \multirow[b]{2}{*}{860} \\
\hline & nigA & $h \lg A-\mathrm{R}$ & TTACTTAGGTGTGATGCTTTTAATTTTTACTTCATGTG & \\
\hline & $h l_{\sigma} R$ & $h \lg B-\mathrm{F}$ & ATGAAAATGAATAAATTAGTCAAATCATCCGTTGC & \multirow{2}{*}{792} \\
\hline & nigh & $h \lg B-\mathrm{R}$ & TTACTGTAATTTTAGATTTTTTAGCGCCATCTTG & \\
\hline & $h l o C$ & $h \lg C-\mathrm{F}$ & GCTTAAAAATAAAATATTAACTACAACTTTATCTGTGAGC & \multirow{2}{*}{720} \\
\hline & mige & $h \lg C-\mathrm{R}$ & TCAATTCTGTCCTTTCACCTTGATTTCATGAG & \\
\hline \multirow{7}{*}{ Leukocidin } & & lukE-F & TTGTCAGTAGGACTGATTGCACCTTTAGC & \multirow{2}{*}{906} \\
\hline & IUKE & $l u k E-\mathrm{R}$ & TTAATTATGTCCTTTCACTTTAATTTCGTGTGTT & \\
\hline & lukM & $l u k M-\mathrm{F}$ & GTTTAAGAGAAAATTATTAGTTACAACTTTGTCGC & \multirow{2}{*}{864} \\
\hline & ІИКМ & $l u k M-\mathrm{R}$ & GTTGTGCCCCTTTACTTTAATTTCGTG & \\
\hline & lukF-PV & $l u k F-P V-\mathrm{F}$ & ATGAAAAAAATAGTCAAATCATCAGTTGTTACATCA & \multirow{2}{*}{978} \\
\hline & & $l u k F-P V-\mathrm{R}$ & AGCTCATAGGATTTTTTTCCTTAGATTGAG & \\
\hline & lukS-PV & $l u k S-P V-\mathrm{F}$ & GGTCAAAAAAAGACTATTAGCTGCAACATTG & 939 \\
\hline \multirow{8}{*}{ Invasive proteases } & $\operatorname{splB}$ & $s p l B-\mathrm{F}$ & GAACAAAAACGTAGTCATCAAGAGTTTAGCAGC & \multirow{2}{*}{723} \\
\hline & & $s p l B-\mathrm{R}$ & CTATGTTTTCTGCAATGAATTTTTTAATTTCTGGTGT & \\
\hline & & $\operatorname{ssp} A-\mathrm{F}$ & ATGAAAGGTAAATTTTTAAAAGTTAGTTCTTTATTCGT & \multirow{2}{*}{783} \\
\hline & $\operatorname{sspA}$ & sspA-R & ATCTTCAATATTTTGTTTTAAGAAGTTGCGTACA & \\
\hline & $\operatorname{ssp} B$ & $\operatorname{ssp} B-\mathrm{F}$ & GTAAATCTAGAGTATTCAATATTATCAGCATCATAATGG & \multirow{2}{*}{792} \\
\hline & & $s s p B-\mathrm{R}$ & AACCTATCATTGAACCATACCAGTTATAATCA & \\
\hline & $\operatorname{ssp} C$ & $\operatorname{ssp} C-\mathrm{F}$ & GTATCAACTACAATTTATAAATTTAGTTTACGACACAACC & \multirow{2}{*}{330} \\
\hline & $\operatorname{ssp} \mathrm{C}$ & $s s p C-\mathrm{R}$ & CTAAGCGCTCATAAACGATTGGGCGC & \\
\hline \multirow{2}{*}{ Hyaluronidase } & $h v s A$ & hys A-F & ATGACATATAGAATGAAGAAATGGCAAAAATTATCCACC & \multirow{2}{*}{936} \\
\hline & hys A & hysA-R & TAATTCAAAGCGCACGCCGGATTCATTAGA & \\
\hline \multirow{2}{*}{ Staphylokinase } & & $s a k-\mathrm{F}$ & ATGCTCAAAAGAGGTTTATTATTTTTAACTGTTTT & \multirow{2}{*}{492} \\
\hline & sak & $s a k-\mathrm{R}$ & ATTTCTTTTCTATAACAACCTTTGTAATTAAGTTG & \\
\hline \multirow{2}{*}{ Lipase } & lin & lip-F & GTAGATTATGGTGCAGCACATGCAGCAAAATATGG & \multirow{2}{*}{921} \\
\hline & $\operatorname{ltp}$ & lip-R & AGCTTTTCAGTTTTCACTAAATCGTCTGCT & \\
\hline \multirow{2}{*}{ Nuclease } & $n u c$ & $n u c-F$ & AAGAGGTTTTTCTTTTTCGCTACTAGTTGC & \multirow{2}{*}{537} \\
\hline & ruc & $n u c-\mathrm{R}$ & CTCCAAATATTTAATTTCTGTTGTTTAGCTTT & \\
\hline
\end{tabular}

TABle 2: Antimicrobial resistance rate of the 33 Staphylococcus aureus strains.

\begin{tabular}{lccc}
\hline Antibiotics & Resistance rate $(n=33)(\%)$ & Intermediary rate $(n=33)(\%)$ & Sensitivity rate $(n=33)(\%)$ \\
\hline Penicillin G & $33(100.00)$ & $0(0.00)$ & $0(0.00)$ \\
Oxacillin & $15(45.45)$ & $0(0.00)$ & $18(54.55)$ \\
Amoxicillin/clavulanic acid & $22(66.67)$ & $0(0.00)$ & $11(33.33)$ \\
Gentamicin & $13(39.39)$ & $0(0.00)$ & $10(30.30)$ \\
Ciprofloxacin & $24(72.73)$ & $0(0.00)$ & $9(27.27)$ \\
Compound sulfamethoxazole & $21(63.64)$ & $0(0.00)$ & $12(36.36)$ \\
Clindamycin & $15(45.45)$ & $0(0.00)$ & $18(54.55)$ \\
Erythrocin & $28(84.85)$ & $0(0.00)$ & $5(15.15)$ \\
Linezolid & $0(0.00)$ & $0(0.00)$ & $33(100.00)$ \\
Teicoplanin & $0(0.00)$ & $0(0.00)$ & $33(100.00)$ \\
Vancomycin & $0(0.00)$ & $0(0.00)$ & $33(100.00)$ \\
Tigecycline & $0(0.00)$ & $0(0.00)$ & $33(100.00)$ \\
Chloramphenicol & $2(6.06)$ & $0(0.00)$ & $31(93.94)$ \\
\hline
\end{tabular}

and 29 all showed a frequency of $94.00 \%(16 / 17)$ and identical gene patterns. The frequency of strain no. 30 showed the lowest value of $29.00 \%$ (5/17). In summary, the six genes encoding hemolysin were found in 29 strains, and the four genes encoding invasive proteases were detected in seven strains. 
TABLE 3: Distribution of virulence genes among MSSA and MRSA strains (\%).

\begin{tabular}{|c|c|c|c|c|}
\hline \multirow[b]{2}{*}{ Virulence genes } & \multicolumn{4}{|c|}{ Frequency } \\
\hline & $\begin{array}{c}\text { Total number of strains }(n=33) \\
(\%)\end{array}$ & $\begin{array}{c}\text { Number of MSSA strains }(n=18) \\
(\%)\end{array}$ & $\begin{array}{c}\text { Number of MRSA strains }(n=15) \\
(\%)\end{array}$ & $P$ value \\
\hline hla & $32(96.97)$ & $17(94.44)$ & $15(100.00)$ & 0.27 \\
\hline$h l b$ & $32(96.97)$ & $17(94.44)$ & $15(100.00)$ & 0.27 \\
\hline hld & $31(93.94)$ & $17(94.44)$ & $14(93.33)$ & 0.89 \\
\hline$h \lg A$ & $29(87.88)$ & $15(83.33)$ & $14(93.33)$ & 0.37 \\
\hline hlgB & $32(96.97)$ & $17(94.44)$ & $15(100.00)$ & 0.27 \\
\hline hlgC & $29(87.88)$ & $15(83.33)$ & $1(6.67)$ & $\leq 0.01$ \\
\hline$l u k-F / S-P V$ & $7(21.21)$ & $1(5.56)$ & $6(40.00)$ & 0.01 \\
\hline$l u k E$ & $17(51.52)$ & $8(44.44)$ & $9(60.00)$ & 0.37 \\
\hline lukM & $7(21.21)$ & $5(27.28)$ & $2(13.33)$ & 0.30 \\
\hline $\operatorname{ssp} A$ & $30(90.91)$ & $16(88.89)$ & $14(93.33)$ & 0.66 \\
\hline$s s p B$ & $29(87.88)$ & $3(16.67)$ & $14(93.33)$ & $\leq 0.01$ \\
\hline$s s p C$ & $27(81.82)$ & $16(88.89)$ & $12(80.00)$ & 0.48 \\
\hline$s p l B$ & $17(51.52)$ & $8(44.44)$ & $9(60.00)$ & 0.37 \\
\hline hysA & $10(30.30)$ & $6(33.33)$ & $4(26.67)$ & 0.68 \\
\hline sak & $23(69.70)$ & $13(72.22)$ & $11(73.33)$ & 0.94 \\
\hline lip & $28(84.85)$ & $17(94.44)$ & $15(100.00)$ & 0.27 \\
\hline nuc & $32(96.97)$ & $13(72.22)$ & $14(93.33)$ & 0.10 \\
\hline
\end{tabular}

TABLE 4: Virulence gene patterns of 33 S. aureus strains.

\begin{tabular}{|c|c|c|c|c|}
\hline $\begin{array}{l}\text { Serial } \\
\text { number }\end{array}$ & Virulence gene patterns & $\begin{array}{c}\text { Strain } \\
\text { number }\end{array}$ & $\begin{array}{c}\text { Number of } \\
\text { genes } \\
(n=17)(\%)\end{array}$ & $\begin{array}{c}\text { Total number of } \\
\text { strains }\end{array}$ \\
\hline 1 & $h l b, h l d, s s p A, s s p B, s s p C, s a k$ & 10 & $6(0.35)$ & 1 \\
\hline 2 & hla, hlgB, sspB, lukM, lip & 15 & $5(0.29)$ & 1 \\
\hline 3 & hla, hlb, hld, hlgB, sspA, sspB, sspC, nuc, lip & 23 & $9(0.53)$ & 1 \\
\hline 4 & hla, hlb, hlgB, sspB, lip & 30 & $5(0.29)$ & 1 \\
\hline 5 & $h l a, h l b, h l d, h l g A, h l g B, h l g C, l u k-F / S-P V, l u k E, s s p A, s s p B, s s p C, s p l B, s a k, n u c, l i p$ & $1,2,3,4,5,6$ & $15(0.88)$ & 6 \\
\hline 6 & $h l a, h l b, h l d, h l g A, h l g B, h l g C, l u k-F / S-P V, l u k E, s s p A, s s p B, s s p C, s p l B, n u c, l i p$ & 7 & $14(0.82)$ & 1 \\
\hline 7 & $h l a, h l b, h l d, h l g A, h l g B, h l g C, \operatorname{ssp} A, \operatorname{ssp} B, s s p C, s a k, n u c, l i p$, hys $A$ & 8,9 & $12(0.71)$ & 2 \\
\hline 8 & $h l a, h l b$, hld, hlgA, hlgB, hlgC, lukM, sspC, splB, sak, lip & 11 & $11(0.65)$ & 1 \\
\hline 9 & $h l a, h l b, h l d, h l g A, h l g B, h l g C, l u k M, \operatorname{ssp} A, \operatorname{ssp} B, s s p C, \operatorname{splB}$, lip & 12 & $12(0.71)$ & 1 \\
\hline 10 & hla, hlb, hld, hlgA, hlgB, hlgC, sspA, sspB, sspC, sak, nuc, lip & $13,26,27$ & $12(0.71)$ & 3 \\
\hline 11 & hla, hlb, hld, hlgA, hlgB, hlgC, sspA, sspB, sspC, splB, lip & 14 & $11(0.65)$ & 1 \\
\hline 12 & hla, hlb, hld, hlgA, hlgB, hlgC, lukE, sspA, sspC, splB, nuc, lip, hys $A$ & 16 & $13(0.76)$ & 1 \\
\hline 13 & $h l a, h l b, h l d, h l g A, h l g B, h l g C, l u k M, s s p A, s s p B, s s p C, s p l B, s a k, n u c, l i p$ & 17 & $14(0.82)$ & 1 \\
\hline 14 & hla, hlb, hld, hlgA, hlgB, hlgC, sspA, sspB, sspC, nuc, lip, hysA & 18 & $12(0.71)$ & 1 \\
\hline 15 & hla, hlb, hld, hlgA, hlgB, hlgC, lukE, lukM, sspA, sspB, sspC, splB, sak, nuc, lip, hys $A$ & $19,20,29$ & $16(0.94)$ & 3 \\
\hline 16 & $h l a, h l b, h l d, h l g A, h \lg B, h l g C, \operatorname{ssp} A, \operatorname{ssp} B, \operatorname{ssp} C, n u c, l i p$ & 21 & $11(0.65)$ & 1 \\
\hline 17 & hla, hlb, hld, hlgA, hlgB, hlgC, sspA, sspB, sak, nuc, lip & $22,31,33$ & $10(0.59)$ & 3 \\
\hline 18 & $h l a, h l b, h l d, h l g A, h l g B, h l g C, \operatorname{ssp} A, \operatorname{ssp} B, \operatorname{ss} p C, s a k, n u c, l i p$, hys $A$ & 24 & $14(0.82)$ & 1 \\
\hline 19 & hla, hlb, hld, hlgA, hlgB, hlgC, sspA, sspC, sak, nuc, lip & 25 & $11(0.65)$ & 1 \\
\hline 20 & $h l a, h l b, h l d, h l g A, h \lg B, h l g C, l u k-F / S-P V, l u k E, s s p A, s s p B, s s p C, s p l B, n u c$, lip & 28 & $14(0.82)$ & 1 \\
\hline 21 & hla, hlb, hld, hlgA, hlgB, hlgC, lukE, lukM, sspA, splB, sak, nuc, lip, hys $A$ & 32 & $14(0.82)$ & 1 \\
\hline
\end{tabular}

3.4. System Cluster Analysis Results. As shown in Figure 1, strain no. 15 and 30 showed high similarity in virulence gene patterns.
3.5. Clinical Data of 33 Patients with S. aureus Infection. All 33 patients with $S$. aureus infections had different basic diseases; 39.39\% (13/33) patients had hypertension, and 


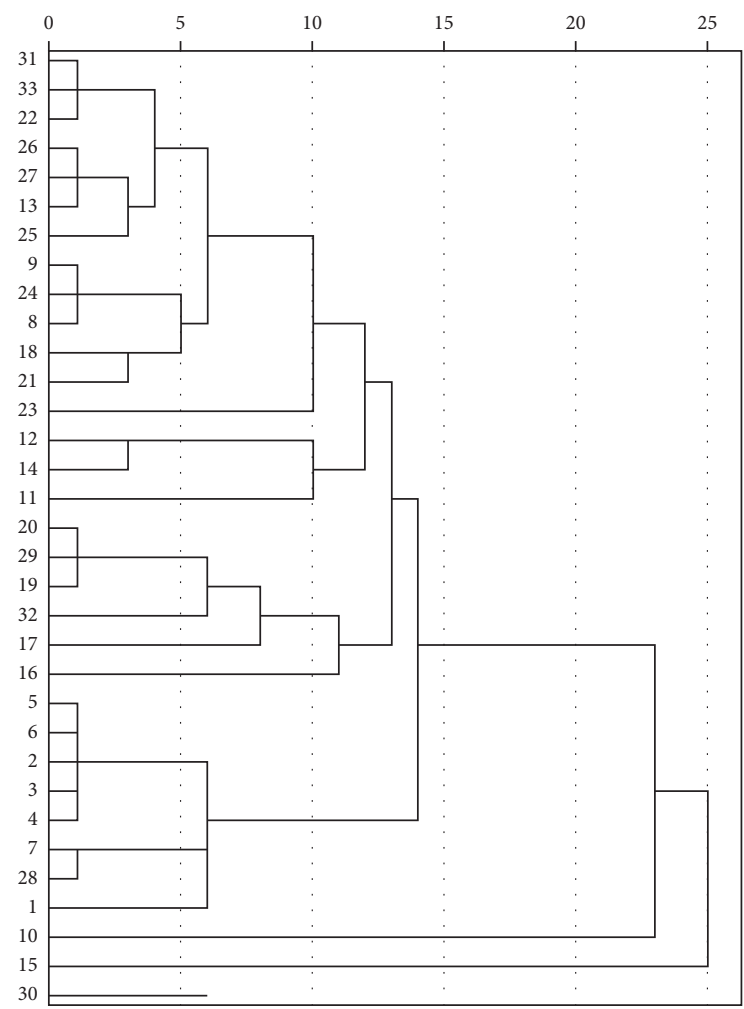

FIGURE 1: System cluster analysis of 33 Staphylococcus aureus virulence genes.

$33.33 \%(11 / 33)$ had trauma and infections (Table 5). A total of $36.36 \%(12 / 33)$ of patients had fever symptoms and invasive channels. Invasive medical procedures were performed in $100.0 \%$ (33/33) of patients. Among them, 24.24\% $(8 / 33)$ and $36.36 \%(12 / 33)$ of patients had undergone percutaneous venous access and placement of drainage tubes, respectively. $9.09 \%(3 / 33)$ and $6.06 \%(2 / 33)$ of patients had been treated to install an indwelling catheter and mechanical ventilation device, respectively. Additionally, 9.09\% (3/33) of patients had concomitant infection with Gram-negative bacilli, including Klebsiella pneumoniae ozaenae and Escherichia coli. We found that $24.24 \%$ (8/33) of patients had been treated with more than three antibiotics. A total of $57.57 \%(19 / 33)$ of patients had a good outcome, and $6.06 \%$ $(2 / 33)$ of the patients died (Table 5$)$.

\section{Discussion}

Invasive $S$. aureus infections are considered as important causes of severe sepsis, with higher mortality rates in developing countries than in developed countries [25]. Monitoring results in recent years have shown that the frequency of MRSA infections has been decreasing each year, both domestically and overseas, whereas the proportion of $S$. aureus has not changed significantly [26-30]. The relationship between $S$. aureus antibiotic resistance and virulence genes and human diseases requires in-depth analysis. In this study, we tested the antibiotic resistance and virulence gene distribution of $33 \mathrm{~S}$. aureus strains isolated from sterile body fluid samples. The results showed that the frequency of MRSA did not significantly differ from that of the MRSA isolated from various specimens in our hospital from 2011 to 2017. Additionally, the rate of resistance to penicillin $G$ was $100.00 \%(33 / 33)$. The resistance rates of these strains to erythromycin and ciprofloxacin were significantly higher than those of strains from three sample types (blood, pus, and secretions) reported by Zheng [8]. The resistance rates to gentamicin and clindamycin were slightly lower. The resistance rate to chloramphenicol was the lowest. No strains resistant to restricted antibiotics, including vancomycin, teicoplanin, linezolid, and tigecycline, were found, suggesting that use of vanguard-restricted antibacterial drugs such as vancomycin will have effective antibacterial effects based on antimicrobial susceptibility tests.

Our results showed that the frequency of $h l a, h l b, h l g B$, and $n u c$ was $96.97 \%(32 / 33)$, which is similar to the results reported by Zhang et al. [31]. All six genes encoding hemolysin were detected in 33 isolates, with the lowest frequency of $87.88 \%$ (29/33). Clinical symptoms and image data showed that seven patients had pneumonia. With respect to hospital infectious pneumonia, there is a significant relationship between bacterial colonization specificity, virulence, clinical outcomes, and mortality [32]. In this study, patients who died or had poor prognosis had pulmonary infection and harbored more than 14 virulence genes. The genes encoding leukocidin showed the lowest frequencies among all virulence genes. The frequency of $l u k E$ was $51.52 \%$ (17/33). The frequency of $l u k-F / S-P V$ was similar to that of the three strains detected in sterile body fluid samples and significantly differed from that of the strains from sputum and wound samples reported in our previous study [20, 21]. The virulence gene carrying rate also showed obvious differences from strains in a group of blood samples abroad $[31,33]$. This may be because different specimen types were used. Genes encoding leukocidin were not detected in 13 strains. None of the 13 patients had clinical symptoms or manifestations of pneumonia, except for the patient carrying strain no. 26. It has been reported that $23 \%$ of medical workers and $18 \%$ of patients are carriers of Staphylococcus. Nasopharyngeal Staphylococcus carriers are typically the main patients with Staphylococcus invasive infections $[34,35]$. The frequencies of $h y s A, s a k$, and lip were different from those in $19 \mathrm{~S}$. aureus strains from blood stream infection in China reported by Zhang et al. [31]. Bacterial virulence genes may be closely related to the evolution of bacteria and host factors $[9,32]$.

We found that the frequencies of $h l a, h l b$, and lip in the 15 MRSA strains were $100 \%(33 / 33)$ and the frequency of $h l g C$ was $6.67 \%(1 / 15)$. Strains carrying $l u k-F / S-P V$ were not detected. The frequencies of $h l a, h l b, h l d, h l g B$, and $l i p$ in the 18 MSSA strains were significantly lower than those in the MRSA strains $(P<0.05)$. The frequency of $h \operatorname{lgC}$ in the MSSA strains was significantly higher than that in the MRSA strains $(P<0.05)$. There was no significant difference in the remaining virulence genes between the MRSA and MSSA strains. The overall virulence gene carrying rate of MSSA was lower than that of MRSA, which differs from the results of previous studies [36-39]. Whether the cause is related to the specimen type, regional differences, and quantity of 
TABLE 5: Clinical data of 33 patients with S. aureus infection.

\begin{tabular}{|c|c|c|}
\hline \multicolumn{2}{|l|}{ Clinical information } & Number of patients $(n=33)(\%)$ \\
\hline \multirow{7}{*}{ Basic disease (7) } & Diabetes & $6(18.18)$ \\
\hline & Hypertension & $7(21.21)$ \\
\hline & Cardiovascular disease & $1(3.03)$ \\
\hline & Uremia & $1(3.03)$ \\
\hline & Trauma & $5(15.15)$ \\
\hline & Respiratory infection & $5(15.15)$ \\
\hline & Urinary tract infection & $1(3.03)$ \\
\hline \multirow{2}{*}{ Sign (2) } & Fever & $23(69.70)$ \\
\hline & Chest film/CT with positive sign & $8(24.24)$ \\
\hline \multirow{4}{*}{ Invasive medical procedures (4) } & Percutaneous venous access & $8(24.24)$ \\
\hline & Drainage tube & $12(36.36)$ \\
\hline & Indwelling catheter & $3(9.09)$ \\
\hline & Mechanical ventilation & $2(6.06)$ \\
\hline Concomitant infection (1) & Gram-negative bacilli infection & $3(9.09)$ \\
\hline \multirow{5}{*}{ Outcome (5) } & Cure & $4(12.12)$ \\
\hline & Improved & $15(45.45)$ \\
\hline & Automatic discharge & $5(15.15)$ \\
\hline & Death & $2(6.06)$ \\
\hline & Other & $2(6.06)$ \\
\hline \multicolumn{2}{|c|}{ Treated with more than three antibiotics } & $8(24.24)$ \\
\hline
\end{tabular}

specimens should be further examined. We performed systematic cluster analysis on the virulence gene patterns of the 33 S. aureus strains, which revealed that the virulence gene patterns of strains no. 15 and 30 were highly similar.

All 33 patients in this study had underlying diseases. All patients had been treated with invasive medical procedures, which is an important cause of infections. The patients were administered more than three types of antibiotics, and most patients infected with MRSA had improved outcomes or were cured. No significant correlation was found between MRSA strain infection and patient death or poor prognosis, which is consistent with previous reports [34, 40]. Studies have reported that $S$. aureus-carrying antibiotic resistance genes may reduce the expression of virulence factors $[10,41]$. We did not detect related antibiotic resistance genes. However, there was no significant difference in the frequencies of major virulence genes between the 18 MSSA strains and 15 MRSA strains. Five patients, including one dead and four unhealed, carried more than 14 virulence genes, indicating that the carrying rate of the virulence genes is related to clinical symptoms and outcomes.

\section{Conclusions}

Staphylococcus aureus strains are highly resistant to conventional antibacterial drugs. Invasive medical procedures are the main causes of infection. Resistance to antibacterial drugs and the status of virulence genes are highly related to clinical symptoms and outcomes. Further studies are needed to determine the mechanisms of virulence genes and their relation to clinical symptoms and outcomes.

\section{Data Availability}

The data used to support the findings of this study are included within the article.

\section{Disclosure}

Tao Lin and Qianhui Li are co-first authors. Chaogui Tang (hayytcg@163.com) and Xiaoyun Zhang (xyzhang2009@ 126.com) are co-corresponding authors.

\section{Conflicts of Interest}

The authors declare no conflicts of interest regarding the publication of this paper.

\section{Authors' Contributions}

Tao Lin and Qianhui Li contributed equally to this work.

\section{Acknowledgments}

This research was funded by the Science and Technology Development Fund of Nanjing Medical University, grant no. 2017NJMU090.

\section{References}

[1] A. D. Wilde, D. J. Snyder, N. E. Putnam et al., "Bacterial hypoxic responses revealed as critical determinants of the host-pathogen outcome by TNSEQ analysis of Staphylococcus aureus invasive infection," PLoS Pathogens, vol. 11, no. 12, Article ID e1005341, 2015.

[2] P. Yoong and V. J. Torres, "The effects of Staphylococcus aureus leukotoxins on the host: cell lysis and beyond," Current Opinion in Microbiology, vol. 16, no. 1, pp. 63-69, 2013.

[3] A. Hamdan-Partida, S. González-García, and J. Bustos Martinez, "Community-acquired methicillin-resistant Staphylococcus aureus can persist in the throat," International Journal of Medical Microbiology, vol. 4, no. 308, pp. 469-475, 2018. 
[4] D. Grumann, U. Nübel, and B. M. Bröker, "Staphylococcus aureus toxins - their functions and genetics," Infection, Genetics and Evolution, vol. 21, pp. 583-592, 2014.

[5] J. M. King, K. Kulhankova, C. S. Stach, and G. Bao Vu, "Phenotypes and Virulence among Staphylococcus aureus USA100, USA200, USA300, USA400, and USA600 Clonal Lineages," mSphere, vol. 1, no. 3, Article ID e00071, 2016.

[6] J. Paulsen, A. Mehl, A. Askim, E. Solligård, B. O. Asvold, and J. K. Damas, "Epidemiology and outcome of Staphylococcus aureus bloodstream infection and sepsis in a Norwegian county 1996-2011: an observational study," BMC Infectious Diseases, vol. 15, no. 116, pp. 116-119, 2015.

[7] W. He, Y. Liu, J. Qi et al., "Food-animal RelatedStaphylococcus aureusMultidrug-resistant ST9 strains with toxin genes," Foodborne Pathogens and Disease, vol. 10, no. 9, pp. 782-788, 2013.

[8] Z. Sun, "Virulence gene detection and drug resistance analysis of Staphylococcus aureus," Journal of Dalian Medical University, vol. 30, pp. 1-45, 2018.

[9] C. He, S. Xu, H. Zhao et al., "Leukotoxin and pyrogenic toxin Superantigen gene backgrounds in bloodstream and wound Staphylococcus aureus isolates from eastern region of China," BMC Infectious Diseases, vol. 18, no. 1, pp. 395-403, 2018.

[10] M. Otto, "Basis of virulence in community-associated methicillin-resistant Staphylococcus aureus," Annual Review of Microbiology, vol. 64, no. 1, pp. 143-162, 2010.

[11] S. Naik, F. Smith, J. Ho et al., "Staphylococcal enterotoxins G and I, a cause of severe but reversible neonatal enteropathy," Clinical Gastroenterology and Hepatology, vol. 6, no. 2, pp. 251-254, 2008.

[12] E. Ghaznavi-Rad, M. N. Shamsudin, Z. Sekawi et al., "Predominance and emergence of clones of hospital-acquired methicillin-resistant Staphylococcus aureus in Malaysia," Journal of Clinical Microbiology, vol. 48, no. 3, pp. 867-872, 2010.

[13] X. Xu, H. Wang, and H. Lu, "Research progress of Staphylococcus hemolysin," Chinese Journal of Microbial, vol. 29, no. 6, pp. 720-724, 2017.

[14] D. Dekker, M. Wolters, and E. Mertens, "Antibiotic resistance and clonal diversity of invasive Staphylococcus aureus in the rural Ashanti RegionGhana," BMC Infectious Diseases, vol. 1, no. 16, Article ID 720, 2016.

[15] B. Löffler, M. Hussain, M. Grundmeier et al., "Staphylococcus aureus panton-valentine leukocidin is a very potent cytotoxic factor for human neutrophils," PLoS Pathogens, vol. 6, no. 15, Article ID e1000715, 2010.

[16] X. Xu, Y. Fan, and S. Hu, "Study on cytotoxin, invasive toxin and capsular antigen gene of Staphylococcus aureus isolates from burn patients," Chinese Journal of General Practice, vol. 14, no. 1, pp. 12-15, 2016.

[17] P. M. Schlievert, K. L. Strandberg, Y.-C. Peterson, and D. Y. M. Leung, "Secreted virulence factor comparison between Methicillin-resistant and Methicillin-Sensitive Staphylococcus aureus, and its relevance to atopic dermatitis," The Journal of Allergy and Clinical Immunology, vol. 125, no. 1, pp. 39-49, 2010.

[18] P. Boan, H. L. Tan, J. Pearson, G Coombs, C. H Heath, and J. O Robinson, "Epidemiological, clinical, outcome and antibiotic susceptibility differences between PVL positive and PVL negative Staphylococcus aureus infections in Western Australia: a case control study," BMC Infectious Diseases, vol. 15, no. 10, pp. 10-16, 2015.

[19] S. Deinhardt-Emmer, S. Sachse, J. Geraci et al., "Virulence patterns of Staphylococcus aureus strains from nasopharyngeal colonization," Journal of Hospital Infection, vol. 100, no. 3, pp. 309-315, 2018.

[20] C. Tang, X. Zhang, and T. Lin, "Study on cytotoxin and invasive toxin genes of Staphylococcus aureus invasive infection isolates," Chinese Journal of Clinical Infected Disease, vol. 7, no. 5, pp. 468-470, 2014.

[21] C. Tang, Y. Chen, and Q. Li, "Study on resistance genes and capsular antigen genes of staphylococcus aureus in invasive infections," Journal of Chinese Hospital Infectious Diseases, vol. 26, no. 7, pp. 1448-1450, 2016.

[22] C. Tang and T. L. Q. Li, "Detection of mecA gene and three virulence factor encoding genes of Staphylococcus aureus," Journal of Chines Hospital Infectious Diseases, vol. 23, no. 24, pp. 5901-5904, 2013.

[23] L. F. Corredor Arias, J. S. Luligo Espinal, and J. I. Moncayo Ortiz, "Relationship between uperantigenicity, antimicrobial resistance and origin of Staphylococcus aureus isolated," Colombia Médica, vol. 47, no. 1, pp. 15-20, 2016.

[24] M. Emaneini, R. Bigverdi, D. Kalantar et al., "Distribution of genes encoding tetracycline resistance and aminoglycoside modifying enzymes in Staphylococcus aureus strains isolated from a burn center," Annals of Burns and Fire Disasters, vol. 26, no. 2, pp. 76-80, 2013.

[25] E. K. Nickerson, V. Wuthiekanun, G. Wongsuvan et al., "Factors predicting and reducing mortality in patients with invasive Staphylococcus aureus disease in a developing country," PloS One, vol. 4, no. 8, Article ID e6512, 2009.

[26] D. Zhu, F. Wang, and F. Hu, "Surveillance of CHINET drug resistance in China in 2010," Chinese Journal of Infection and Chemotherapy, vol. 11, no. 5, pp. 321-329, 2011.

[27] F. Hu, Y. Guo, and D. Zhu, "CHINET bacterial resistance monitoring results in 2016," Chinese Journal of Infection and Chemotherapy, vol. 17, no. 5, pp. 481-490, 2017.

[28] A. El-Ghodban, K. S. Ghenghesh, K. Márialigeti, H. Esahli, and A. Tawil, "PCR detection of toxic shock syndrome toxin of Staphylococcus aureus from Tripoli, Libya," Journal of Medical Microbiology, vol. 55, no. 2, pp. 179-182, 2006.

[29] J. Walter, I. Noll, M. Feig et al., "Decline in the proportion of methicillin resistance among Staphylococcus aureus isolates from non-invasive samples and in outpatient settings, and changes in the co-resistance profiles: an analysis of data collected within the Antimicrobial Resistance Surveillance Network, Germany 2010 to 2015," BMC Infectious Diseases, vol. 17, no. 1, p. 169, 2017.

[30] C. Mottola, T. Semedo-Lemsaddek, J. J. Mendes et al., "Molecular typing, virulence traits and antimicrobial resistance of diabetic foot staphylococci," Journal of Biomedical Science, vol. 23, no. 1, p. 33, 2016.

[31] Q. Zhang, H. Ma, and Y. Zhang, "Study on virulence genes of methicillin-resistant Staphylococcus aureus blood isolates," Chinese Journal of Infectious Disease, vol. 26, no. 21, pp. 4859-4862, 2016.

[32] H. Sedaghat, B. N. Esfahani, M. Halaji et al., "Genetic diversity of Staphylococcus aureus strains from a teaching hospital in Isfahan, Iran: the emergence of MRSA ST639- SCCmec III and ST343- SCCmec III," Iranian Journal of Microbiology, vol. 10, no. 2, pp. 82-89, 2018.

[33] D. Eibach, M. Nagel, B. Hogan et al., "Nasal carriage of Staphylococcus aureus among children in the ashanti region of Ghana," PLoS One, vol. 12, no. 1, Article ID e0170320, 2017.

[34] C. Kebaier, R. R. Chamberland, I. C. Allen et al., "Staphylococcus aureus $\alpha$-hemolysin mediates virulence in a murine model of severe pneumonia through activation of the NLRP3 
inflammasome," The Journal of Infectious Diseases, vol. 205, no. 5, pp. 807-817, 2012.

[35] H. R. Rose, R. S. Holzman, D. R. Altman et al., "Cytotoxic virulence predicts mortality in nosocomial pneumonia due to methicillin-ResistantStaphylococcus aureus," Journal of Infectious Diseases, vol. 211, no. 12, pp. 1862-1874, 2015.

[36] J. K. Rudkin, M. Laabei, A. M. Edwards et al., "Oxacillin alters the toxin expression profile of community-associated methicillin-resistant Staphylococcus aureus," Antimicrobial Agents and Chemotherapy, vol. 58, no. 2, pp. 1100-1107, 2014.

[37] J. Wang, X. Du, and L. Ta, "Methicillin-resistant/sensitive Staphylococcus aureus genotyping and virulence gene detection," Chinese Journal of Infection and Chemotherapy, vol. 15, no. 1, pp. 70-74, 2015.

[38] J. Tong and Z. Zhan, "Staphylococcus aureus virulence gene detection and molecular typing," Chinese Journal of Microbiology and Immunology, vol. 35, no. 1, pp. 46-48, 2015.

[39] R. Köck, K. Winner, F. Schaumburg, A. Jurke, J. W. Rossen, and A. W. Friedrich, "Admission prevalence and acquisition of nasal carriage of meticillin-resistant Staphylococcus aureus (MRSA) in German rehabilitation centres," Journal of Hospital Infection, vol. 87, no. 2, pp. 115-118, 2014.

[40] D. A. Williamson, S. R. Ritchie, S. A. Roberts et al., "Clinical and molecular epidemiology of community-onset invasive Staphylococcus aureus infection in New Zealand children," Epidemiology and Infection, vol. 142, no. 8, pp. 1713-1721, 2014.

[41] J. Collins, J. Rudkin, M. Recker, C. Pozzi, J. P. O’Gara, and R. C. Massey, "Offsetting virulence and antibiotic resistance costs by MRSA," The ISME Journal, vol. 4, no. 4, pp. 577-584, 2010 . 